ACTUALIZACIÓN EN

\title{
Evaluación de competencias del proceso formativo de los residentes utili- zando el portafolio: una revisión de la literatura
}

\author{
[Assessment of competencies of the residents' training process using the portfolio: a literature review] \\ Dra. Virginia E. Díaz A ${ }^{1}$ \\ 'Pediatra Neumóloga. Hospital del Niño Dr. José Renán Esquivel. Panamá, República de Panamá.
}

Correspondencia:Dra.VirginiaE.DíazA.Correo electrónico:vedadiaz@gmail.com

Recibido: 25 de marzo, 2020

Aceptado: 30 de marzo, 2020

Publicado: 15 de octubre, 2020

Cita: Díaz VE, (2020), Evaluación de competencias del proceso formativo de los residentes utilizando el portafolio: una revisión de la literatura. DOI: $10.37980 /$ im.journal.rspp2019.1593

Palabras clave: educación médica, tutores, portafolio, aprendizaje reflexivo, evaluación de las competencias clínicas.

Keywords: medical education, tutors, portfolio, reflective learning, evaluation of clinical skills.

Reproducción: Artículo de acceso libre para uso personal e individual. Sujeto a derechos de reproducción para otros usos.

Conflictos de interés: Los autores declaran no tener conflictos de interés.

Financiamiento: La autora no declara fuentes externas de financiamiento asociados a este trabajo.

\section{Resumen}

En el proceso dinámico de los cambios y mejoras curriculares en las diferentes especialidades se han tomado en cuenta los conocimientos, habilidades, actitudes y valores; por eso tienen que integrarse nuevas formas de estudio, supervisión y evaluación de los médicos en formación. Experiencias recientes en nuestro país tratan de introducir los portafolios profesionales como herramientas de evaluación-aprendizaje en la formación especializada de posgrado. El portafolio es un instrumento de evaluación del proceso formativo de los residentes que permite no sólo evaluar las competencias, sino fundamentalmente identificar en qué debemos mejorar permitiendo dar seguimiento a los medios en formación según vayan avanzando en la adquisición y desarrollo de competencias de manera que se convierta en una herramienta enfocada a ayudarnos a aprender y aporta, al mismo tiempo, elementos para valorar la participación de los tutores y la influencia de los mismos en los educandos.En el proceso de planeación, diseño y revisión de los portafolios los centros formadores encuentran una excelente oportunidad de hacer una autoevaluación de la congruencia de su estructura curricular, de la claridad de las competencias que busca formar y de las evidencias que puede exigir en la formación de sus residentes.

\section{Abstract}

In the dynamic process of curricular changes and improvements in the different specialties, knowledge, skills, attitudes and values have been taken into account; that is why new forms of study, supervision and evaluation of doctors in training have to be integrated. Recent experiences in our country try to introduce professional portfolios as assessment-learning tools in specialized postgraduate training. The portfolio is an instrument for evaluating the residents' training process that allows not only evaluating competencies, but fundamentally identifying what we should improve, allowing us to monitor the training media as they advance in the acquisition and development of competencies so that become a tool focused on helping us learn and at the same time provide elements to value the participation of tutors and their influence on learners. In the process of planning, designing and reviewing the portfolios, the training centers find an excellent opportunity to make a self-assessment of the consistency of their curricular structure, the clarity of the competences they seek to form and the evidence they may require in the training of its residents.

\section{Introducción}

Los puntos de vista sobre cómo evaluar la competencia médica y el rendimiento están cambiando por lo que conceptos como evaluación formativa, evaluación de la práctica clínica, evaluación clínica objetiva estructurada entre otros están más presentes en la práctica de la medicina, tanto en la esfera clínico-asistencial como en las tareas docentes y de tutoría de los médicos en formación. ${ }^{1,2}$
En el proceso dinámico de las mejoras curriculares en las diferentes especialidades se han tomado en cuenta los conocimientos, habilidades, actitudes y valores; por eso tienen que integrarse, de la misma manera, nuevas formas de estudio, supervisión y evaluación de los médicos en formación

La educación basada en competencias destaca la importancia de la información continua al alumno sobre el rendi- 
miento; por lo tanto, la retroalimentación formativa se convierte en un componente necesario de la evaluación de las competencias. ${ }^{3}$ El aprendizaje reflexivo es la clave para desarrollo profesional. ${ }^{9}$ Parboosingh ${ }^{10}$ considera el componente esencial del aprendizaje como la capacidad de práctica de cambio como resultado del aprendizaje. Por lo tanto, es necesario que el alumno reflexione sobre las necesidades de aprendizaje, la necesidad a través de actividades de aprendizaje, y luego reflexionar sobre cómo este aprendizaje afectará la práctica futura.

El portafolio es una "colección de pruebas mantenidas y presentadas para un propósito específico".4 El uso del portafolio amplía el alcance de la evaluación al abarcar una variedad de documentos en papel, audio o formato digital que pueden demostrar el logro de la competencia del residente. ${ }^{5}$

El portafolio refuerza la formación a través del propio proceso evaluativo por lo que es una herramienta con un elevado impacto educativo que intensifica las interacciones tutor-residente, al proporcionar material de discusión, asegurar la dedicación de un tiempo específico a la reflexión sobre el aprendizaje, establecer la bidireccionalidad del aprendizaje, estimular al tutor en el planteamiento de estrategias creativas de aprendizaje y favorecer la retroalimentación del binomio tutor-residente. ${ }^{5}$

\section{Discusión}

El portafolio es un instrumento fundamental de evaluación que consiste en la recopilación de la información, en distintos formatos, con los que el profesional demuestra su desarrollo profesional y la adquisición de un determinado nivel de competencia, que ya existía o que se ha alcanzado durante el proceso de evaluación. ${ }^{6}$

Sirve como instrumento de evaluación formativa y sumativa porque permite compilar diversos métodos de evaluación, generando un documento oficial del desempeño del residente durante sus años de formación.

Las evidencias que muestren la progresión en el aprendizaje del residente se pueden encontrar en cualquiera de los productos de sus actividades como los registros clínicos, las evaluaciones externas, informes de rotaciones, etc., o en tareas específicamente diseñadas para exponer determinados aspectos de la práctica tales como los análisis de incidentes críticos y resúmenes de casos entre otros. ${ }^{5}$

El portafolio es un ejercicio de autodisciplina que consiste en la práctica de una reflexión permanente sobre los problemas que surgen en nuestro trabajo diario. Se trata de anotar y de registrar de una forma estructurada estas situaciones que nos permiten aprender $y$, una vez anotadas, obligarnos a pensar qué podemos aprender, de qué manera y con qué mecanismos, a fin de resolver el problema de aprendizaje que haya surgido e incrementar así nuestra pericia profesional. ${ }^{7}$
Fig. 1. Pirámide de Miller. Relación entre nivel de competencia y los instrumentos susceptibles de ser utilizados. Adaptada de: MiIler GE. The assessment of clinical skills/competence/performance. Academic Medicine. 1990;65 (Suppl): S63-7.

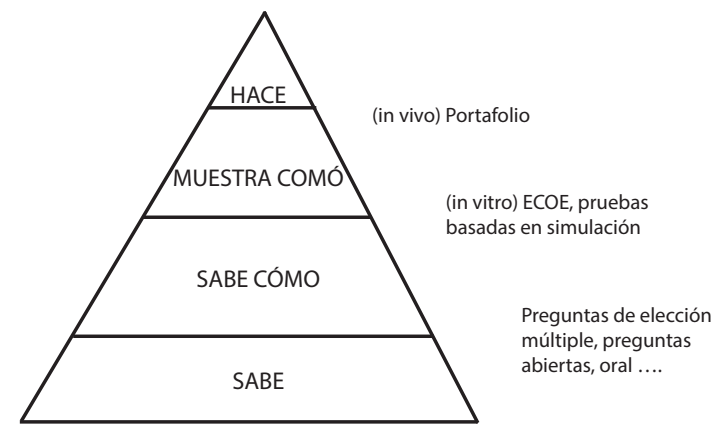

La pirámide de Miller (Figura 1) establece 4 niveles de aprendizaje con sus respectivos niveles de evaluación de competencias. En el nivel más básico, el conocer se aplica la evaluación de los conocimientos, es decir la capacidad para recordar cosas; en el segundo nivel, el cómo conocer se evalúa la capacidad para entender cómo ocurren los fenómenos es decir, para buscar información analizarla e interpretar resultados; en el tercer nivel, el cómo mostrar, se evalúa la ejecución de lo que se ha aprendido, es decir, implica una acción, pero en una práctica in vitro, no en condiciones de práctica real en el vértice de la pirámide está el hacer que se evalúa en la práctica real, in vivo. ${ }^{8}$ El portafolio está ubicado en el vértice de la pirámide de Miller porque permite analizar aquello que el profesional hace, más que los conocimientos o aquello que es capaz de hacer, en su práctica real y proporciona información complementaria que permite que quien lo utiliza deje constancia de sus experiencias de aprendizaje, su capacidad para plantear y resolver preguntas sobre la práctica, la progresión en la resolución de problemas, las actitudes ante los dilemas que plantea el ejercicio profesional, la inquietud para investigar y el hábito de planificar el crecimiento personal y profesional mediante la formulación de objetivos propios de formación y mejora. ${ }^{5,7}$

El uso del portafolio se inició a mediados de la década de 1980, por artistas, arquitectos, fotógrafos, diseñadores, etc. El portafolio, como construcción procedente de las artes plásticas, pretende recoger las pruebas demostrativas de las habilidades y técnicas que su autor ha ido desarrollando, en un área o período de tiempo determinados, con la finalidad de dar a conocer su obra. ${ }^{7,9,10}$ Paulson ${ }^{11}$ afirma que un portafolio es una colección de trabajos del estudiante que refleja el progreso, esfuerzo y logros del individuo. Judith $\operatorname{Arter}^{12}$ destaca que el portafolio electrónico es una herramienta para tener evidencia del desarrollo del individuo, evalúa sus competencias y permite motivar e involucrar al estudiante en su propio aprendizaje. 
Su propósito fundamental, es la demostración de avances en un proceso de formación. El portafolio como una herramienta en el proceso de la enseñanza en la medicina permite al residente reflexionar sobre el aprendizaje que ha obtenido, lo que en sí mismo modifica la percepción de la experiencia vivida, permitiendo integrar lo aprendido al conocimiento previo y utilizarlo para iniciar un nuevo aprendizaje y promoviendo la evaluación basada en el desempeño y haciendo posible el seguimiento a los alumnos según vayan avanzando en el desarrollo de las competencias. ${ }^{10}$

Kolb ${ }^{13}$ exploró el principio del aprendizaje por medio de la experiencia describiéndolo como un ciclo que, explícitamente, incorpora y construye sobre las experiencias de las que se deriva el aprendizaje. El ciclo incluye las etapas de una experiencia definida, reflexionando sobre la misma para de ahí plantear generalizaciones que puedan aplicarse en otras situaciones, y probarlas a través de una nueva experiencia, con un sentido de progresión. Para realizar esto es necesario contar con cuatro habilidades: tener una experiencia concreta; realizar una observación reflexiva; conceptualizar o deducir generalizaciones abstractas y aplicar los conocimientos a nuevas situaciones. Stanton ${ }^{14}$ afirma que el aprendizaje basado en el portafolio tiene sus raíces en los principios de aprendizaje basado en experiencias.

La construcción del portafolio requiere que los documentos que se incorporen apoyen y confirmen el proceso de reflexión y aprendizaje que ha motivado el análisis de dichos elementos: ¿Qué he aprendido? A través de la identificación de experiencias, importantes fuentes de aprendizaje; ¿Cómo lo he aprendido? Mediante la especificación de cómo ese aprendizaje es demostrado en la práctica; ¿Cómo lo he aplicado? Es decir qué hago bien o mejor ahora por la identificación de qué aprendizaje fue obtenido a través de la experiencia; ¿Qué me falta por aprender o reforzar y qué haré para aprenderlo? Al identificar las necesidades adicionales de aprendizaje medios por los que estas pueden cumplirse. ${ }^{5,10}$

Como objetivo de aprendizaje, el portafolio estimula el aprendizaje integrado, auto-reflexivo, autodirigido, cooperativo y longitudinal. Como objetivo de evaluación permite reflexionar sobre el trabajo realizado, la retroalimentación recibida, el progreso logrado y los planes para mejorar la competencia que se desea alcanzar. ${ }^{15}$

El portafolio debe ser flexible y debe adaptarse a las características personales del residente y al año de residencia. Debe basarse en la práctica asistencial cotidiana y promover la práctica reflexiva permanente. Los documentos almacenados deben proporcionar evidencias del progreso en el aprendizaje y del nivel alcanzado y debe estar centrado en los objetivos del aprendizaje, establecidos en el programa de formación de la especialidad para cada año de la residencia. ${ }^{8}$
El tutor tiene funciones importantes en la implementación y desarrollo del portafolio. Las más importantes y fundamentales son: explicar al residente la filosofía del instrumento, su implementación práctica y el manejo de esta herramienta; ayudar a elaborar el portafolio, a resolver dudas y dificultades y supervisar la construcción del mismo, sugerir líneas o temas de reflexión y apoyar el proceso reflexivo del residente, registrar por escrito y transmitir al residente las consideraciones pertinentes sobre cada uno de los componentes del portafolio y proporcionar retroalimentación. ${ }^{8}$

El portafolio parte del análisis de la propia práctica del residente, pero la figura del tutor es imprescindible. El uso del portafolio permite también valorar la participación de los funcionarios tutores y su influencia en la educación del residente. Este instrumento evidencia el compromiso del tutor en la planificación del aprendizaje, ya que proporciona el espacio para identificar problemas de aprendizaje, promover en el residente la reflexión sobre dichos problemas, el seguimiento de la adquisición y la aplicación de conocimientos y habilidades y, finalmente, la planificación de la formación del residente. 5,15

La aplicación del portafolio en el proceso de aprendizaje del residente se ha incorporado en varias instituciones que forman residentes en diferentes especialidades. ${ }^{6,7,16-18}$ Las conclusiones de estos estudios se citan a continuación. Los resultados orientan a una buena acogida por los residentes y los tutores. En el proceso de implementación, las sugerencias $y$ aportaciones de unos y otros permiten corregir, y especialmente simplificar, una herramienta que debe aunar eficacia y sencillez en su manejo si quiere cumplir su objetivo. Esta herramienta proporciona un avance y una contribución interesante en el proceso formativo del residente y en la tarea docente del tutor. ${ }^{16}$ El portafolio es una herramienta que los residentes implementan con sus tutores y ambos colectivos la perciben como útil para reflexionar sobre la práctica y para fortalecer su relación. ${ }^{17}$

En un estudio realizado ${ }^{7}$ los resultados coinciden con los obtenidos en otros estudios citados por Ruiz, ${ }^{19}$ en los que se concluye que para que esta metodología sea aceptada se deben cumplir una serie de premisas como son: la importancia clave de una buena relación tutor-residente, que los tutores estén bien entrenados, y una cuidadosa planificación de su introducción y puesta en marcha. La introducción de este instrumento formativo en la tutorización de residentes puede ser considerada de interés para tutores y residentes, identificándose como una de sus principales fortalezas la sistematización de un aprendizaje reflexivo a partir de la propia práctica y el ser un marco para la facilitación y fortalecimiento de la relación tutor-residente. ${ }^{8}$

El portafolio proporciona una herramienta para recolectar y administrar múltiples tipos de evidencia de evaluación de 
múltiples contextos y fuentes dentro del plan de estudios para documentar la competencia y promover habilidades de práctica reflexiva. ${ }^{18}$

\section{Comentarios finales}

El portafolio es una colección de evidencias de los productos y procesos de aprendizaje, que evidencia la adquisición de competencias y mediante el análisis crítico de sus contenidos, el desarrollo personal y profesional del futuro especialista.

El portafolio aporta al proceso de tutorización del residente elementos novedosos como: el valor del análisis de la propia práctica como fuente inagotable de aprendizaje porque permite asociar la propia experiencia a la interiorización del aprendizaje; el hábito de aportar pruebas, tareas o evidencias, que muestran la consecución de objetivos formativos; la responsabilidad activa en su propia formación, centrada en las necesidades del residente en cada momento de su trayectoria; la fundamentación los modelos de aprendizaje de los adultos y la base para desarrollar una interrelación y comunicación entre el tutor y el residente encaminada a la excelencia profesional.

El portafolio cumple varios propósitos fundamentales:

- Identifica experiencias significativas: importantes fuentes de aprendizaje.

- Identifica qué aprendizaje se obtuvo a través de la experiencia y cómo ese aprendizaje es demostrado en la práctica.

- Identifica, reconocer y mejorar los procesos de aprendizaje

- Inicia al residente procesos de auto reflexión, conductas éticas y atención con calidez

- Orienta la relación de tutorización hacia una relación de confianza y respeto mutuo.

- Promueve hábitos de responsabilidad, de planificación de su formación: autoaprendizaje por elección

- Estimula la creatividad y promueve valores y actitudes éticas.

- Integra la información clínica relevante a las ciencias básicas o viceversa y a las nuevas situaciones.

- Permite la evaluación basada en desempeño, posibilitando dar seguimiento a los residentes, según vayan avanzando en el desarrollo de competencias.
En resumen, compila:

- ¿Qué aprendí? La identificación de experiencias como importantes fuentes importantes de aprendizaje.

- ¿Qué hago bien o mejor ahora? La identificación de qué aprendizaje fue obtenido a través de la experiencia.

- ¿Cómo lo aprendí? La especificación de cómo ese aprendizaje es demostrado en la práctica.

- ¿Qué me faltó por aprender o reforzar y cómo lo logro? La identificación de necesidades adicionales de aprendizaje y de los medios por los que éstas pueden cumplirse

Propongo incorporar el portafolio en la evaluación de competencias del proceso formativo de los residentes.

\section{Referencias}

1. Asociación de Redes de Comisiones de Docencia y Asesoras (AREDA). La evaluación de la formación especializada como garantía de la calidad del sistema de salud. Educ Méd. 2007;10(1):16-25.

2. Epstein RM. Assessment in Medical Education. N Engl J Med. 2007;356: 387-396. doi:10.1056/NEJMra054784

3. Challis M. AMEE medical education guide no. 11 (revised): Portfolio-based learning and assessment in medical education. Med. Teacher. $1999 ; 4: 437-440$.

4. Mathers NJ, Challis MC, Howe AC, Field NJ. Portfolios in continuing medical Education - Effective and efficient? Med. Education.1999;33:521-30.doi:10.1046/j.1365-2923.1999.00407.x

5. Torán-Monserrat P, Arnau i Figueras J. El portafolio como instrumento de valoración del residente. Aten. Primaria. 2006; 37(7):371-3. doi: 10.1157/13087367.

6. Saura Llamas J, Martínez Garre MN, Sebastián Delgado ME et al. Evaluación formativa mediante el portafolio de 4 promociones de residentes de medicina de familia y comunitaria de la Unidad Docente de Murcia. Aten. Primaria. 2013;45(6):297-306. doi.org/10.1016/j.aprim.2012.12.004

7. Arnau i Figueras J, Torán Monserrat $P$, Martínez-Carretero JM, Forteza-Rey J, Pinilla Llorente B, Brailovsky CA. Introducción del portafolio formativo como instrumento de tutorización de residentes de Medicina Interna: revisión de una experiencia piloto, 2005-2006. Rev Clin Esp. 2008; 208(9):447-54. doi. org/10.1157/13127606

8. Grupo de Trabajo Portafolio SEMI. Guía de Trabajo Portafolio SEMI: Curso académico 2007-08. Sociedad Española de Medicina Interna-Institut d'Estudis de la Salut. Disponible en: http:// www.fesemi.org/cuaderno/public

9. Nolla-Domenjó M. La evaluación en educación médica. Principios básicos. Educ. Méd. 2009;12(4):223-229

10. Rodríguez WF, Pedraza ML. Fundamentos para la implantación del portafolio académico como otro elemento en la evaluación del residente. Med Int Mex. 2009;25(5):393-394.

11. Paulson FL, Paulson PR, Meyer CA. What makes a portfolio a 
portfolio? Educational Leadership. 1991;48(5):60-63.

12. Arter JA. Using Portfolios in Institution and Assessment: State of the Art Summary. Portland, Oregon. Norwest Regional Edu-17. cational Laboratory. November 1990. 1-43

13. Kolb DA. Structural Foundations of the Learning Process. Experiential Learning.Experience as the Source of Learning and Development, Glasser (Ed) Pearson Education, Inc. New Jersey, USA, 2nd Edition, 2014:65-66

14. Stanton F, Grant J. Approaches to experimental learning,18. course delivery and validation in medicine. A background document. Med. Educ. 1999;33(4):282-97. doi:10.1046/j.13652923.1999.00272.x

15. Casanova JM, Soria X, Borrego L, de Argila D, Ribera M, Pujol19. R. El portafolio como herramienta de formación y evaluación de los residentes de Dermatología (II). Actas Dermosifilogr. 2011;102(5):325-335. doi:10.1016/j.ad.2010.11.003

16. Schlatter J. Experiencias del uso del portafolio para residentes de la Clínica Universidad de Navarra. www.fundacionedu- caionmedica.org FEM 2013;16(1):59-62. dx.doi.org/10.4321/ S2014-98322013000100010

Moral Ruiz R, Alba Dios A, Pérula De Torres LA et al.El portafolio del libro del especialista en Medicina de Familia en formación: grado de implementación y opiniones de residentes y tutores 2 años después de su implantación en una unidad docente. Aten Primaria.2009;41(11):607-612. doi.org/10.1016/j. aprim.2009.02.006

Dannefer EF, Henson LC. The portfolio approach to competency-based assessment at the Cleveland Clinic Lerner College of Medicine. Acad Med. 2007; 82(5):493-502. doi:10.1097/ACM. Ob013e31803ead30

Ruiz R. Opiniones de tutores y residentes tras aplicar un sistema de evaluación formativa tipo portafolio: la nueva propuesta de «Guía de práctica reflexiva» del Libro del especialista en Medicina de Familia en formación. Educ Med. 2008;11 (3):147155. Disponible en: http://scielo.isciii.es/scielo.php?script=sci_arttext\&pid=S1575-18132008000300007\&lng=es. 